\title{
L'INTELLECTUEL, PROVISOIREMENT, LÉGITIME: FRANÇOIS MAURIAC ET LE DISCOURS RELIGIEUX DU POLITIQUE
}

\author{
Helenice Rodrigues da Silva \\ Chercheur-associé: Laboratoire "Communication et Politique" (CNRS, Paris). \\ Professeur visitant Depto. de História-FFLCH/USP, São Paulo
}

RESUMO: A posição e a "disposição" (o "habitus") de F. Mauriac no campo intelectual françês não o predispunha à uma designação de intelectual "legítimo" (engajado e da esquerda). No entanto, a sua trajetória, nos anos 50, na qualidade de jornalista, dentro da revista L'Express, atestam novas posições politícas, condizentes com à forma cultural dominante. Se, durante os últimos anos da IV República, Mauriac se mobiliza politicamente em defesa dos oprimidos na África do Norte e, sobretudo, se engaja moralmente no combate à tortura, a partir da $V^{a}$ República, no entanto, essa consciência cristã engajada se afastará do combate político. E isso em razão mesmo de sua adesão total e infalível à pessoa do general de Gaulle.

ABSTRACT: François Mauriac's position and "disposition" ("habitus") in the French intellectual field does not predispose him to be designated as a "legitimate" intellectual (committed and on the left). However, his trajectory in the 50's, as a journalist working in L'Express bears witness to his new political positions, much more in harmony with the dominant cultural model. If, during the last years of the Fourth Republic, Mauriac takes political actions in order to stand up for the oppressed people from North Africa, and above all, morally commits himself to the fight gainst torture, this committed Christian conscience nonetheless withdraws from the political fight from the start of the Fifth Republic. It can be attributed to his complete support of General de Gaulle.

PALAVRAS-CHAVE: engajamento, habitus, campo intelectual, anticolonialismo, gaullismo.

KEY WORDS: commitment, habitus, intellectual field, anticolonialism, gaullism.

\section{Parcours et choix politiques}

La position que François Mauriac occupe dans le "champ" intellectuel français des années 50 est pour le moins singulière. Intellectuel catholique engagé, il n'appartient ni à la gauche chrétienne ni à 
la génération intellectuelle issue de la Résistance.

Sa pensée fortement imprégnée de catholicisme demeure inséparable de sa condition même d'intellectuel. De la lecture de ses Bloc-Notes ${ }^{1}$ par exemple, se dégage une dimension de foi chrétienne qui sert de cadre référentiel à son langage. Cet écrivain, déjà consacré, se servira du journalisme (tout au long des années 50) comme d'une tribune privilégiée pour ses interventions politiques. Le rapport des intellectuels avec l'espace public correspond, d'ailleurs, à une pratique courante dans ces années de l'après-guerre. En effet, on peut retracer l'évolution de sa pensée politique en suivant son parcours dans diverses revues et publications de l'époque. Les différents champs de production, on le verra, seront déterminants pour l'élaboration de divers types de discours.

Ainsi, sa présence au Figaro ${ }^{2}$ et à La Table Ronde correspond-elle à un Mauriac, "plus ou moins maurrassien du début des années 50" (LACOUTURE, 1980, p.160), défenseur de la colonisation française et du "génie national français". Pendant la guerre d'Indochine, il s'oppose farouchement à toute possibilité de négociations avec le Vietminh et justifie la présence française au nom d'une "civilisation bienfaisante". Ces prises de position s'expliquent par son anticommunisme manifeste formulé à maintes reprises à

${ }^{1}$ Écrits entre 1952 et 1964, ils furent édités en trois volumes: 1) 1952-1957; 2) 1958-1960; 3) 1961-1964. "... Quelqu'un est là, avec ses idées, ses goûts, ses humeurs, les conditions d'une vie ordinaire, et chaque semaine il réagit à l'Histoire telle qu'elle se fait sous son regard. Cet affrontement de l'individuel et de l'universel, c'est tout le Bloc-Notes..." (MAURIAC, Bloc-Notes, 1952-1957, Flammarion, 1958, p.3)

${ }^{2}$ En qualité de journaliste, François Mauriac écrira régulièrement pour ce quotidien de 1934 à 1954, sans interrompre pour autant sa collaboration au Figaro Littéraire.

3 "Il ressort de mes éditoriaux du Figaro que je croyais l'époque ${ }^{3}$. Au demeurant, Mauriac considère la colonisation comme une sorte de Croisade et comme telle, celle-ci pouvait être équivoque. D'après lui, les violences perpétrées dans le système colonial ne font que traduire "la corruption d'une grande idée"4.

Néanmoins, la crise marocaine de $1953^{5}$ provoque chez Mauriac une profonde émotion et marque la rupture d'une certaine façon de penser la politique. Sa première réaction est celle d'un chrétien indigné par la répression qui sévit au Maroc contre les fidèles de l'Islam. Le caractère religieux et non communiste de ce mouvement nationaliste explique en grande partie son adhésion à la cause marocaine. "Au risque d'être dupe, je crois pourtant que c'est l'heure ou jamais, au Maroc, d'une politique généreuse, c'està-dire confiante et fraternelle. Une protestation de la conscience chrétienne, l'Islam, à notre insu, l'attendait. Entre toutes les sottises criminelles qui ont cours aujourd'hui dans certains milieux, la plus criminelle et la plus sotte est l'opposition Orient-Occident, Chrétienté-Islam". (MAURIAC, 1967, d, p.455) C'est donc en tant qu'humaniste chrétien qu'il prend d'abord position, dans les colonnes du Figaro, contre les injustices et les brutalités commises par l'administration française à l'égard des musulmans d'outre-mer.

très possible sinon probable, une invasion de l'Europe par l'armée rouge". (MAURIAC, Mémoires Politiques, Grasset, 1967, p.30)

${ }^{4}$ Cité par Les Temps Modernes, mars 1947, "Indochine SOS", p.1041.

${ }^{5}$ Les émeutes des nationalistes musulmans furent provoquées par le départ du sultan Mohammed Ben Youssef. Ce dernier, confronté à un mouvement hostile à sa personne et à la politique d'Istiqlal céda. Derrière le sultan, le mouvement des "Jeunes Marocains" et le parti Istiqlal demandaient la fin du protectorat et l'établissement d'une monarchie constitutionnelle. 
Nous retrouvons, éternellement vivant, écrit-il, une exigence de justice, et si indignes que nous soyons, la nécessité de rendre témoignage, dans l'intervalle de temps et de l'espace où nous sommes au monde. (MAURIAC, 1967, d, p.458).

Mauriac s'engage ${ }^{6} d^{\prime}$ autant plus à fond dans la campagne pro-marocaine que des intellectuels chrétiens anticolonialistes ${ }^{7}$ lui demandent de prêter sa notoriété à la cause des nationalistes marocains. L'espace de son action va ainsi dépasser le champ même des publications. Il se joint à d'autres intellectuels catholiques, et ensemble, ils feront du Centre Catholique des Intellectuels Français (CCIF) un autre pôle de combat de l'intelligentsia chrétienne contre le colonialisme en Afrique du Nord. Plutôt qu'une institution à caractère religieux, le CCIF fut notamment un lieu de dialogue ouvert aux intellectuels, en principe proches du catholicisme. A partir d'une vision "humaniste-chrétienne", ses adeptes s'interrogeaient sur les problèmes politiques les plus pressants de l'époque.

Dans le but de mieux informer l'opinion française sur l'évolution de la situation marocaine, Mauriac crée en 1953 le Comité "France-Maghreb". Un bulletin mensuel d'information (sur les événements qui se déroulaient au Maroc et en Tunisie) voit le jour à la fin de cette même année.

\footnotetext{
${ }^{6}$ Lors de sa mobilisation en faveur des nationalistes marocains, Mauriac s'explique à propos de l'engagment de l'écrivain: "De nombreux écrivains se jugent eux-mêmes invités par vocation à ne se mêler que de l'éternel. Je l'ai cru moi-même, à certaines époques. Mais notre génération a payé cher cette connaissance que nous sommes tous engagés, que nous sommes tous embarqués dans la même tragique aventure, tous solidaires, tous responsables, et que de tous les partis pris, le refus de prendre parti est sans doute le seul qui ne puisse trouver, ni devant Dieu ni devant les hommes, de justification"... (Ibid., p.458)

${ }^{7}$ cf. LACOUTURE, J. François Mauriac. Seuil, 1980, p.446.
}

La mobilisation de Mauriac, selon ses propres mots, "prend forme, se développe, sans fièvre, dans la paix et la certitude" (MAURIAC, 1958, a, p.3), en faveur du retour du sultan au Maroc. "Le sultan religieusement et politiquement, non seulement au Maroc, mais dans tout l'islam n'incarnait-il pas la force spirituelle qu'il ne fallait à aucun prix rabaisser ni humilier?" (MAURIAC,J., 1958, a, p.45), s'interroget-il. Au demeurant, la déposition du sultan pendant la Fête du Sacrifice, fut ressentie comme un sacrilège et comme un affront pour les croyants de l'islam. Donc, au-delà d'un coup de force politique, il s'agissait bien, selon Mauriac, d'une manoeuvre religieuse voire d'une violence symbolique commise contre l'orthodoxie musulmane.

C'est sans doute dans les colonnes de Témoignage Chrétien et dans celles de $L^{\prime}$ Express $^{8}$ (à partir de novembre 1953) que Mauriac va exprimer, en toute liberté, ses nouvelles positions politiques. Dans l'impossibilité de concilier ses idées anticolonialistes avec les tendances politiques des publications telles que La Table Ronde, il en retire ses Bloc-Notes à la fin de l'année 1953 et les place dans L'Express.

Cette rupture correspond pour lui à une volonté nette de se démarquer d'une certaine mouvance politique de droite, hostile à ses nouvelles positions anticolonialistes ${ }^{9}$. Son adhésion à l'équipe de L'Express

\footnotetext{
${ }^{8}$ L'objectif de cet hebdomadaire de centre-gauche (qui devient quotidien d'octobre 1955 à mars 1956 pour mieux lutter aux côtés de Mendès France) étant plutôt de véhiculer les idées politiques de Pierre Mendès France et de le porter au pouvoir. Quant à Témoignage Chrétien, de 1954 à 1956, cette publication exprime par le biais d'un catholicisme de gauche un ensemble de positions inspirées par le mendésisme.

${ }^{9}$ Il va sans dire que ses articles dérangent à la fois une partie de l'opinion publique conservatrice et les organes de presse hostiles aux événements nationalistes d'outre-mer.
} 
et son ralliement au "mendésisme"10 le force à quitter le Figaro en octobre 1954, lorsque celui-ci attaque P. Mendès France ${ }^{11}$. En s'affiliant politiquement au mouvement "mendésiste" et en se plaçant aux côtés des intellectuels de gauche anticolonialistes, autrement dit en s'engageant explicitement et symboliquement dans un combat politique, il semble subir des pressions du champ intellectuel de l'époque, largement marqué à gauche. Ainsi, partageant provisoirement un habitus conforme, Mauriac n'adhère-t-il pas au rang d'intellectuel "légitime"?

Il reste, cependant, à préciser les contours de l'engagement et à définir les modalités de l'anticolonialisme chez Mauriac. C'est par rapport à la religion et à la morale qu'il pense le "système de domination nationale". "A l'ensemble des chrétiens (...) de l'Union Française et de la Métropole s'impose le devoir de faire front contre ce racisme né du lucre et de la peur, qui enfante les crimes collectifs" (LACOUTURE, 1980, p.456) ${ }^{12}$. Il va sans dire que son anticolonialisme s'apparente à celui des humanistes en général, qui, parce qu'ils ont "une certaine idée de la France", voire une irréfutable croyance en sa "mission civilisatrice", prônent plus de justice et exigent par conséquent des réformes dans les colonies. Ils ne songent pas pour autant à la séparation

\footnotetext{
${ }^{10}$ Son attitude est représentative du ralliement de certains intellectuels catholiques à P. Mendès France lors des guerres coloniales. Ce courant politique, aux contours souvent changeants et imprécis, a connu l'adhésion d'une nouvelle génération de la gauche universitaire (composée notamment par des normaliens), déçue par les partis politiques de la gauche traditionnelle: la SFIO agonisante et le PCF imprégné de stalinisme.

${ }^{11}$ Elu Président du Conseil (juin 1954/mars 1955), Pierre Mendès France inaugure un nouveau style en politique. En
}

politique (l'indépendance nationale), comme ce sera d'ailleurs le cas lors du conflit algérien.

L'emploi de thèmes récurrents dans le discours de Mauriac, tels que: morale, justice, charité, espérance entend bien produire un effet de discours de vérité. "La politique n'échappe pas à la morale, écrit-il. (...) En Indochine, au Maroc, en Tunisie, la vérité politique, c'est la justice". (MAURIAC, 1958, a, p.85.) On ne saurait nier cette forme de vérité, voire d'absolu qui symbolise la foi chrétienne chez Mauriac et qui fonctionne comme socle d'une pensée intellectuelle engagée. De même, ses discours sont empreints d'un caractère hautement prophétique.

\section{Le cri et le silence sur la torture.}

Lorsque l'insurrection algérienne éclate (novembre 1954), il en parle le lendemain même dans son Bloc-Notes, en date du 02/11/54 et l'intitule: "La guerre d'Algérie commence". Dans ce même article, il mentionne déjà la pratique de la torture. "Coûte que coûte, écrit-il, il faut empêcher la police de torturer" (MAURIAC, 1958, a, p.139). Certes, cette question avait été ébauchée antérieurement par des journalistes catholiques dans France-Observateur; il n'empêche qu'elle deviendra très tôt dans L'Express l'enjeu principal du combat de Mauriac.

minorant le rôle des partis politiques, il se fait investir sur son seul programme. Contrariant les normes de la IVème République qui considère que le chef du gouvernement n'a de comptes à rendre qu'aux assemblées, il décide d'expliquer sa politique au pays dans des causeries hebdomadaires radiodiffusées, ce qui le popularise auprès de l'opinion publique.

12 "La vocation des chrétiens dans 1'Union Française" cité par LACOUTURE,J. Le texte constitue le premier de la série de ses écrits engagés. 
C'est dans une chronique datant du 14/1/55 que Mauriac énonce en toutes lettres cette méthode abjecte de répression.

"- Vous seul pouvez parler (...) Mes ennemis croient que je cède à la passion d'occuper la scène. Je soupire:

- Il faudrait des preuves. On n'a jamais de preuves.

- Moi, j'ai vu, dit l'homme (...)

- A quoi bon, puisque "ça" ne laisse pas de traces!

- Ils n'ont pas renoncé aux coups de nerfs de boeuf, vous savez! Mais la baignoire, ou plutôt le baquet d'eau sale où la tête est maintenue jusqu'à l'étouffement, mais le courant électrique (...)

- Ce n'est pas possible, dis-je (...) Je sais... d'autres m'ont raconté. Mais pourquoi? Pourquoi?

- Il s'agit d'obtenir des suspects (...), l'aveu de leur participation directe ou indirecte au terrorisme. Mais surtout, on attend d'eux qu'ils dénoncent leurs camarades (...)

- Les bourreaux n'auront donc même pas l'excuse des conquérants, car ce n'est pas par la force, c'est par son message humain que la France reste conquérante: en la déshonorant, ils la désarment" (MAURIAC, 1958, a, p.146.).

L'auteur de ces lignes a choisi ici la forme discursive d'un dialogue pour traiter d'un sujet à la fois grave et interdit ${ }^{13}$ afin de faire de son énoncé une sorte de je parle, il parle, ça parle. Le référent, c'est à dire le sujet dont on parle - la torture - n'apparaît pas nommé dans ce texte où les mots parlent d'euxmêmes. L'auteur met ainsi en scène une situation apparemment invisible par le biais de la représentation codifiée de la pratique de la torture. Il reviendra sur cette question à d'autres moments.

${ }^{13}$ Il écrira dans ses Bloc-Notes du 08/12/54: “Je n'ose ici rapporter ce que me confiait un avocat d'Alger il y a quelques jours. Ce qu'est le racisme policier en Afrique du Nord, jusqu'à quels excès il se porte. Mais on a honte (...). Le témoignage des victimes ne compte guère. Mais la pudeur me retient" (p.146).
A l'occasion de la clôture de la Semaine des Intellectuels Catholiques, le chrétien Mauriac s'inspire des "bourreaux du Christ"14 pour formuler un de ses discours les plus mystiques contre la torture.

... Le rapport historique entre les peuples dominateurs et les peuples dominés n'a pas sensiblement changé depuis le Christ. (...) Les richesses naturelles que les peuples primitifs détenaient à leur insu ont déchaîné et déchaînent encore une convoitise chez les nations chrétiennes qui, pour s'assouvir, ont répandu et répandent encore beaucoup de sang. Leur domination s'est perpétuée par des procédés qui témoignent ce qui n'est pas l'imitation de Jésus Christ, au cours de l'Histoire, qui est devenue trop souvent la règle de l'occident chrétien. (...) Quelque soit nos raisons de nos excuses, je dis qu'après 19 siècles de christianisme, le Christ n'apparaît jamais dans le supplicié aux yeux des bourreaux d'aujourd'hui, la Sainte Face ne se révèle jamais dans la figure de cet Arabe sur laquelle le commissaire abat son poing. (...)"C'est à moi que vous le faites!" Cette voix qui retentira un jour (...): "J'étais ce jeune homme qui aimait sa patrie et voulait forcer à trahir son frère". Comment cette grâce n'est-elle jamais donnée à aucun bourreau baptisé? (...) A quel moment de l'Histoire (...), les nations chrétiennes ont-elles témoigné qu'elles se souvenaient que le Christ a été un homme torturé dans son corps?..." (LACOUTURE \& MORANDAIS, 1984, pp.19-21)

La force de ce discours tient dans son aspect éminemment symbolique: le bourreau de Christ renvoie à la condition de dominant et le Christ lui-même aux dominés. L'auteur de ces mots fait parler le per-

14 Allocution prononcée le 13/11/54. MAURIAC,F. L'Imitation des Bourreaux de Jésus Christ et présentation des Blocs-Notes sur la torture par LACOUTURE,J. et MORANDAIS, Alain de la, Desclée de Brouwer, 1984. 
sonnage muet: le torturé, le "rebelle", celui qui n'a pas droit à la parole mais qui parle ici par la voix interposée du Christ. Le "sujet énonciateur" ne s'efface pas pour autant pour laisser parler l'autre. Il parle ("je dis que"), il laisse parler une situation symbolique de domination, sans jamais mentionner explicitement le mot "colonisation". C'est par la référence à la religion que l'auteur pense la politique, par l'analogie aux bourreaux du Christ qu'il fait allusion aux tortionnaires, par l'imitation de la voix du Christ qu'il exprime le silence des victimes.

Cette conscience chrétienne, aussi déterminante soit-elle quant aux prises de position politiques de Mauriac, a-t-elle orienté et justifié tous ses engagements?

De 1953 jusqu'au début 1958, Mauriac occupe le devant de la scène politique par son action déterminée contre les abus de la colonisation, contre les injustices coloniales et contre la torture. Il participe en fait à toute forme symbolique de protestation intellectuelle: meetings, manifestations, signatures de pétitions, etc...

Pendant toute cette période, François Mauriac reste, en fait, très critique vis-à-vis de la politique menée par les divers gouvernements qui se succèdent, à l'exception, bien entendu, de celui de Pierre Mendès France en qui il met de grands espoirs de changement. Il s'en prend tour à tour à la droite en général et au MRP en particulier ainsi qu'à la SFIO et à Guy Mollet (Président socialiste du Conseil) qui lui inspirent un assez grand mépris. Mauriac accuse les socialistes de faire la politique de leurs adversaires et d'être ainsi responsables, au même titre que la droite, de l'aggravation de la guerre en Algérie. “...Je me considère, écrit-il, comme au service des Français et des Françaises qui vomissent les paroles menteuses des politiciens (...). Mon rôle à moi est de déranger l'interprétation officielle des événements..." (MAURIAC, 1958, a, pp.213/214) ${ }^{15}$. De surcroît, il dénonce dès le début de la publication des ses Bloc-Notes la crise que traverse l'Etat français pendant la IVème République.

La phrase qui s'ensuit est, quant à elle, placée sous le signe d'un "patriotisme mystique" et d'une fidélité inconditionnelle à la personne du général de Gaulle. Elle marque la fin de ses combats politiques. Le "13 mai 1958" (date à laquelle de Gaulle arrive au pouvoir) déclenche chez Mauriac des ruptures profondes: l'intellectuel engagé d'antan devient un "observateur" qui s'engage à défendre sans réserve la politique gaulliste.

Mais revenons un instant sur la question de la torture. Pour Mauriac, la torture nuit moralement et spirituellement à l'idée de la France et de sa vocation; elle est donc contre-productive pour la pacification. Tout en ayant condamné violemment cette "méthode", Mauriac n'a jamais remis en cause le principe de la pacification en lui-même. (pacification est le nom qui sert à remplacer dans le discours officiel la politique de la guerre). “...Il faut, dit-il, convaincre ce peuple (les Algériens) de notre double résolution: ne pas nous séparer de lui, mais lui rendre dans tous les ordres ce qui lui est dû..." (MAURIAC, 1958, a, p.238). En d'autres termes, Mauriac exprime la pensée des adeptes de la politique des réformes et du fédéralisme, comme solution plausible au conflit algérien.

Dans ses articles sur la torture, l'effet de miroir est présent dans l'argumentation: la nation qui torture devient à son tour la nation torturée, le bourreau devient la victime. La France, terre des droits de l'homme, dégrade son image lorsqu'elle pratique des actes qui nuisent à cette image. Les figures de la victime et du bourreau renvoient à une seule et même

15 Créé au lendemain de la guerre, le MRP (Mouvement Républicain Populaire) bénéficia du soutien d'une grande partie de l'électorat modéré privé de ses partis politiques habituels. La SFIO (parti socialiste), reconstitué pendant la guerre, resta indissociable de son véritable inspirateur, Léon Blum. 
image. Sur ce point, Mauriac argumente de la même manière que Sartre: l'identité est ici dévoilée par l'altérité ${ }^{16}$.

...Notre vocation, c'est de rendre la France ressemblante à cette image qui suivit dans le coeur des hommes libres du monde entier - image vivante qui, elle aussi, subit la torture, chaque fois que quelqu'un est torturé par l'un des nôtres, mais qui y survit parce qu'elle est immortelle... (MAURIAC,1958, a, p.361).

L'ambivalence des positions de Mauriac durant la guerre d'Algérie contraste avec la fermeté et la clarté de ses opinions tout au long de l'affaire marocaine dont la nature, il est vrai, n'est en rien comparable à la situation algérienne ${ }^{17}$. Au fil des années, la lecture des Bloc-Notes permet de déceler les signes de ses contradictions, de ses hésitations ${ }^{18}$ et laisse transparaître son silence sur la question (la torture), une fois de Gaulle au pouvoir.

Cependant, comme le dira Mauriac lui-même: "...L'honneur du journaliste, contrairement à ce que l'on peut penser, consiste davantage à se soucier de dire ce qui lui semble juste au moment où il le dit qu'à ne pas se contredire"... (LACOUTURE, \& MORANDAIS, p.67/ 68). En réalité, il s'agit de savoir qui se contredit: le journaliste ou l'intellectuel?

16 "Si rien ne protège une nation contre elle-même, ni son passé, ni ses fidélités, ni ses propres lois, s’il suffit de quinze ans pour changer en bourreaux les victimes (...). Victimes et bourreaux ne font plus qu'une seule image: et c'est notre image. Dans les deux cas extrêmes, en effet, la seule façon de refuser l'un des deux rôles, c'est de revendiquer l'autre".. (SARTRE, JP. Situations V, 1964, pp.74/75).

${ }^{17}$ Jean Lacouture dans François Mauriac essaie de justifier son "retrait" relatif et ses positions ambivalentes en mettant en avant la force que le mythe "Algérie française" a représenté pour lui durant toute sa vie.
Alors que la torture continue d'être pratiquée en Algérie après l'avènement de la Vème République, que les ratissages et les attentats ${ }^{19}$ sont perpétrés de part et d'autre, de plus en plus ouvertement aussi bien en France qu'en Algérie, les jugements moraux de Mauriac semblent être dictés plutôt par sa fidélité au général de Gaulle et au crédit illimité qu'il lui accorde que par sa "conscience chrétienne". Pour quelqu'un qui pense que le "combat spirituel dans une démocratie se mène conjointement au combat politique", n'est-ce-pas là une contradiction?

Interrogé sur la raison de son silence à propos de l'usage de la torture dans la Vème République, Mauriac répond d'une façon qui n'est guère probante “qu' aucun gouvernement n'a rien pu contre la torture. (...) Pas plus que le Dieu de Malebranche, dit-il, le général de Gaulle n'agit par des volontés particulières; il détruira la torture en détruisant la guerre, si du moins il l'emporte contre la conjuration de ceux qui à Alger et au Caire ont intérêt à ce que la guerre ne finisse pas (...). Un homme seul, peut-il mater les puissances qui s'opposent en Algérie?”... (LACOUTURE,J.; MORANDAIS, A. (de la), p.82). Son argumentation reflète à ce moment-là surtout le fatalisme et le doute. En fait, la vérité chez Mauriac n'est pas toujours synonyme de certitude.

18 “...Je me reproche, écrit Mauriac, le 27/5/62, d'avoir toujours mis l'accent avec trop de complaisance sur la disproportion des deux communautés, d'avoir eu sans cesse à la bouche ces huit cent mille et ces neuf millions, et de ne pas avoir suffisamment tenu compte du dynamisme des moins nombreux et du capital humain qu'ils représentaient" (Nouveau Bloc-Notes; 1961-1964, p.154).

${ }^{19}$ On ne trouve dans ces chroniques aucune référence ni à la violence qui a suivi la manifestation des Algériens en octobre 1961, ni au massacre du métro Charonne en février 1962 qui fit huit morts. 


\section{Le discours de la "grâce"}

Dans les Bloc-Notes écrits entre 1958 et 1960, le thème de la grâce apparaît de manière pertinente. Lorsqu'il est employé dans un contexte politique, c'est en tant que référence symbolique à la personne de de Gaulle.

...La grâce, dans son action pénétrant l'Histoire de la France, trouve son sujet de prédilection dans un homme qui n'est pas l'homme du destin mais "l'homme de la grâce", l'élu de Dieu pour sauver la nation. Chrétien, je me suis senti, quant à moi, confirmé dans ma certitude: Charles de Gaulle n'est pas l'homme du destin, il est l'homme de la grâce... (LACOUTURE, J. p.82).

L'usage de ce langage, fortement codé par la référence à la religion et à la patrie, tend à conférer à son discours des propriétés spécifiques, à la fois mythiques et prophétiques. Son Bloc-Notes daté du 11/5/ 56 traduisait déjà cette forme de sacralisation de de Gaulle:

...Le général de Gaulle, n'a jamais cessé d'incarner pour moi une espérance. Dans notre malheur présent, cette espérance échappe au vague (...). Il m'apparaît comme le seul Français détenteur d'une gloire (...) pour susciter en Afrique, autour de la France, une fédération de peuples libres... (MAURIAC,1958, a, p.235).

Or, la position du général de Gaulle sur la question algérienne revêt une forme pour le moins ambiguë. Revenu au pouvoir grâce au soulèvement et aux appels des "ultras" (ardents partisans de l'Algérie française) à Alger, il était cependant perçu par l'opinion publique métropolitaine et par la majorité de la classe politique comme étant le seul homme capable de sortir l'Algérie de l'impasse. En effet, la Vème République est née du constat d'échec et d'impuissance de la IVème République.

Si de Gaulle était convaincu, comme il l'affirmera dans ses Mémoires d'Espoir (1970) que l'Algérie fran- çaise correspondait en 1958 à une "ruineuse utopie" et l'indépendance à une affaire inéluctable, il laisse cependant planer l'équivoque sur ses intentions réelles. D'autant que, pendant plus d'un an, il jouera le jeu des partisans de l'intégration, c'est-à-dire de l'Algérie française.

L'acte fondateur de ses contradictions futures et, par conséquent de la radicalisation des actions des ultras réside sans doute dans la célèbre phrase qu'il prononça à Alger en juin 1958: "Je vous ai compris!".

En somme, la politique algérienne de de Gaulle semble ne pas avoir obéi à un dessein cohérent qui eût visé à conduire l'Algérie, à court terme, à une indépendance future. Au contraire, les combats s'intensifièrent sous la Vème République. Et il est légitime de se demander si la situation politique ne s'est dénouée que grâce à la pression internationale ainsi qu'à une opposition interne.

A partir du moment où Mauriac se rallie à de Gaulle et par conséquent se démarque de la gauche, il commence à accoler le terme "de gauche" à celui d'"intellectuel". Il dénonce désormais "l'activisme de la presse de gauche" en faisant preuve de beaucoup d'empressement ${ }^{20}$. Une des réactions les plus éloquentes de Mauriac c'est, en 1961, son départ de L'Express pour rejoindre le Figaro Littéraire qui devient dès lors la tribune de ses Bloc-Notes. Il va de soi que son retour à son champ de publication d'antan correspond à ses nouvelles dispositions liées à sa nouvelle prise de position politique.

La déclaration des 121 (manifeste des intellectuels contre la guerre d'Algérie et pour le droit à l'insoumission) et les réactions qu'elle suscite en France et à l'étranger obligent l'intellectuel Mauriac à sortir de sa réserve et à s'exprimer. Il saisit cette occasion pour formuler ses conceptions sur la notion

${ }^{20}$ Bloc-Notes, 18 et 21 mai 1962. 
même de l'intellectuel. Il en profite pour prendre ses distances vis-à-vis des intellectuels qui, selon lui, sont désormais identifiés aux penseurs de gauche. Hostile aux signataires du manifeste, il s'en prend violemment dans cet article aux intellectuels étrangers qui leur sont solidaires.

“... Je me pose la question: suis-je moi-même un intellectuel? Si pour prétendre à ce nom, il faut provoquer et publier (...) des protestations étrangères (...), eh bien! non, je ne me sens rien de commun avec cette espèce-là. (...) Le parti des intellectuels...Pauvres intellectuels, qui prétendez vous dresser dans le monde entier pour défendre le droit à l'insoumission et qui êtes les plus soumis de tous les hommes. (...) Les écrivains, continue-til, décident de tout dans l'absolu. Arrêter la guerre d'Algérie, c'est pour le penseur une opération de la pensée"... (MAURIAC, 1961, b, p.17).

Il prend prétexte de cet article pour réaffirmer sa confiance en "ce général à deux étoiles", le seul capable d'arrêter la guerre. Mais, il clôt ce texte d'une façon inattendue, voire surprenante; en critiquant la déshumanisation de l'homme, il réhabilite alors l'intellectuel. “...Il est vrai que vous demeurez la dernière chance d'une pensée humaine et libre. Pour finir, je vous rends les armes, je vous donne raison"... (MAURIAC, 1968,c, pp.19 et 21).

En l'occurrence, ses Bloc-Notes de la fin de la guerre d'Algérie reflètent sa position défensive face à quelques intellectuels de gauche qui l'accusent de garder le silence. Dans sa chronique du 5/2/62, Mauriac remet en question la notion même d'engagement: réaction d'ailleurs fort symptomatique de sa nouvelle conception de l'intellectuel. "...S'engager, prendre parti, c'est en politique s'exposer à pêcher contre la justice quand nous y pensons le moins, et contre la charité", rappelle-t-il à la fin du conflit algérien. (MAURIAC, 1968, p.157)

En guise de conclusion, l'on pourra dire que l'ébauche même du parcours et du choix d'action de cet intellectuel catholique anticolonialiste doté d'un capital de notoriété, témoigne de la pluralité des perceptions, voire de l'hétérogénéité des synthèses du mouvement anticolonialiste français. Si pour les uns, être anticolonialiste signifie lutter pour l'implantation à la fois d'une politique de réformes et d'un système fédéraliste dans les colonies, pour les autres, en revanche, l'anticolonialisme sou-tend l'idée-même d'une fin du système; autrement dit l'indépendance politique comme seule solution valable.

Or, quelques constatations se dégagent de cette esquisse:

Premièrement: Il est évident que la mouvance anticolonialiste dépasse la sphère symbolique droite/ gauche, tout au moins dans les premières années du conflit algérien.

Deuxièmement: Le souci de "dire la vérité" détermine toutes les positions politiques en cette époque de crise, bien que l'acception du terme "vérité" soit diamétralement variable chez les intellectuels engagés.

Troisièmement: Dans la mesure où le pouvoir politique s'avère incapable de préserver les pratiques démocratiques, l'action des intellectuels devient alors manifeste. Les intellectuels étant censés être les garants de la démocratie, la question se pose alors de savoir jusqu'à quel point leurs actions ont pris la forme symbolique d'un contre-pouvoir provisoire.

Quatrièmement: C'est toujours en fonction d'une conception idéaliste de la France - dont la représentation se fait de façon très différente chez les différents intellectuels - que les notoriétés intellectuelles puisent leurs discours et leurs actions contre la guerre d'Algérie. C'est pour défendre une image de la France ternie par cette guerre que François Mauriac, et notamment Raymond Aron, justifient leur action politique. C'est pour combattre une même image que Sartre, pour sa part, s'investit dans l'action. Il reste que l'efficacité symbolique de l'intervention de François Mauriac sur le terrain politique provient directement 
de son "capital de reconnaissance" à l'intérieur même du champ intellectuel. Or, son engagement politique lors de la décolonisation en Afrique du Nord a renforcé l'image même de cet intellectuel en tant qu'intellectuel.

\section{Bibliographie}

BEDARIDA, François et RIOUX Jean-Pierre. (direct.). Pierre Mendès France et le Mendésisme. Paris, Fayard, 1985. LACOUTURE, Jean. François Mauriac. Paris, Seuil, 1980.

LACOUTURE, Jean et MORANDAIS, Alain de la. L'Imitation des Bourreaux de Christ et présentation des Bloc-Notes sur la torture. Paris, Desclée de Brouwer, 1984.

RIOUX, J.P. et SIRINELLI, J.F. (édité). La Guerre d'Algérie et les Intellectuels Français. Cahier n¹0, IHTP, CNRS, novembre 1988.

MAURIAC, François. Blocs-Notes 1952 - 1957. Paris, Flammarion, 1958, a. Blocs-Notes 1958 - 1960. Paris, Flammarion, 1961, b. Blocs-Notes 1961 - 1964. Paris, Flammarion, 1968, c. Mémoires Politiques. Paris, Grasset, 1967, d.

RUDELLE, Odile. "Gaullisme et crise d'identité républicaine". La Guerre d'Algérie et les Français. Paris, Fayard, 1990. SORUM, Paul Clay. Intellectuals and Decolonization in France. University of North Carolina Press, Chapel Hill, 1977.

TAVARES, Jean. "Le Centre Catholique des Intellectuels Français. Le dialogue comme négociation symbolique". Actes de la Recherche en Sciences Sociales. Paris, $\mathrm{n}^{\circ} 38$, mai 1981.

THIBAUD, Paul. "Génération algérienne?". Esprit, Paris, mai 1990. "Une fidélité têtue: la résistance française à la guerre d'Algérie". Paris, Vingtième Siècle, n 10, 1986

VIDAL-NAQUET, Pierre. Face à la Raison d'Etat - un historien dans la guerre d'Algérie. Paris, La Découverte/ essais, 1989. 